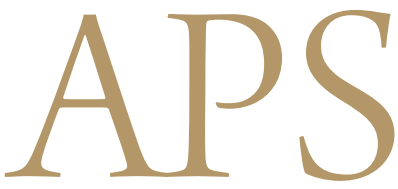

Archives of Plastic Surgery

\title{
A Novel Nipple Reconstruction Technique for Maintaining Nipple Projection: The Boomerang Flap
}

\author{
Young-Eun Kim, Ki Yong Hong, Kyung Won Minn, Ung Sik Jin \\ Department of Plastic and Reconstructive Surgery, Seoul National University Hospital, Seoul National University College of Medicine, Seoul, \\ Korea
}

Nipple-areolar complex (NAC) reconstruction is the final step in the long journey of breast reconstruction for mastectomy patients. Successful NAC reconstruction depends on the use of appropriate surgical techniques that are simple and reliable. To date, numerous techniques have been used for nipple reconstruction, including contralateral nipple sharing and various local flaps. Recently, it has been common to utilize local flaps. However, the most common nipple reconstruction problem encountered with local flaps is the loss of nipple projection; there can be approximately 50\% projection loss in reconstructed nipples over long-term follow-up. Several factors might contribute to nipple projection loss, and we tried to overcome these factors by performing nipple reconstructions using a boomerang flap technique, which is a modified C-V flap that utilizes the previous mastectomy scar to maintain long-term nipple projection.

Keywords Breast reconstruction / Nipple reconstruction
Correspondence: Ung Sik Jin Department of Plastic and Reconstructive Surgery, Seoul National University Hospital, Seoul National University College of Medicine, 101 Daehak-ro, Jongno-gu, Seoul 03080, Korea

Tel: +82-2-2072-2370

Fax: +82-2-3675-7792

E-mail: usj1011@snu.ac.kr

This article contains Supplemental Video S1.

No potential conflict of interest relevant to this article was reported.

Received: 4 May 2016 • Revised: 20 Jul 2016• Accepted: 26 Jul 2016

pISSN: 2234-6163 • elSSN: 2234-6171 • http://dx.doi.org/10.5999/aps.2016.43.5.470 • Arch Plast Surg 2016;43:470-473

\section{INTRODUCTION}

Nipple-areolar complex (NAC) reconstruction is the final procedure of breast reconstruction for breast cancer patients, completing the aesthetic breast. Several methods have been used for NAC reconstruction, including nipple sharing, full-thickness skin grafting, autologous cartilage grafting, and various local flaps $[1,2]$. For successful nipple reconstruction, the method should be uncomplicated and reliable. Moreover, the aesthetics of the nipple shape and the maintenance of long-term nipple projection are important factors for consideration.

Recently, nipple reconstruction has been performed mainly with local flaps, because of low donor site morbidity and rela- tively satisfactory aesthetic outcomes. One of the most commonly utilized methods is the $\mathrm{C}-\mathrm{V}$ flap, and a report evaluating patient satisfaction on long-term follow-up showed $81 \%$ overall satisfaction at an average of 5.3 years of follow-up [3]. However, the most frequently encountered complication in nipple reconstruction is the loss of nipple projection. It has been estimated that approximately $50 \%$ to $70 \%$ projection loss occurs over the long-term follow-up period [4].

Several factors might contribute to nipple projection loss. However, the absence of support at the nipple base, the utilization of a friable and thin dermal flap, and the lack of firm filling material may be the most relevant factors. We have striven to find a reliable method to improve long-term nipple projection that ame- 
liorates those conditions. This report introduces the details of a new technique for nipple reconstruction to improve nipple projection.

\section{IDEA}

The boomerang flap, a modified $\mathrm{C}-\mathrm{V}$ flap method, is composed of two wings, eliminating the $\mathrm{C}$ flap in a C-V flap (Fig. 1). An imaginary ellipse is drawn at the proposed nipple position with the expected immediate postoperative nipple projection (a) and width (b). The immediate postoperative nipple is designed to have $1.5 \times$ higher projection and $1.2 \times$ greater width than the contralateral side due to the expected potential shrinkage during the follow-up period. Each wing forms the pillar and the lid of the nipple. The width of the wing that will form the lid is set to correspond to the expected lid width (c) and it tapers off at the end, while the other wing, forming the pillar, is designed to be longer than the length needed for the circumference of the nipple base $(b+e)$. The indentation (d) is designed as a back-cut for rotation.

The designed flap is elevated and rolled, and 3 key sutures are applied so that 1 wing of the flap forms the pillar and the other wing forms the lid of the nipple. The distal parts are de-epithelialized and packed into the nipple for core volume augmentation (Supplemental Video 1).

By eliminating the $\mathrm{C}$ flap, unlike the $\mathrm{C}-\mathrm{V}$ flap, and using 1 wing to form the lid, the nipple base becomes narrower compared to the top of the nipple. The cross-section of the reconstructed

\section{Fig. 1. The design of the boomerang flap}

(A) An imaginary ellipse is drawn at the proposed neo-nipple position, with its horizontal axis representing the nipple width, and its vertical axis representing the nipple height. Then, the design is made along the long surgical scar, positioning the scar inside the width of the flap. a, nipple height; $b$, nipple base width; c, nipple lid width; $d$, back-cut for rotation; e, nipple base circumference. (B) One of the 2 lateral wings is turned to form the pillar and another wing, instead of a C flap, forms the lid of the nipple; (C) 3 key-sutures are required. (D) The distal parts of the wings are de-epithelialized and packed into the nipple to augment the core volume. (E) The final cross-section of the nipple forms an upside-down trapezoid with the base of the nipple pillar being narrower than the upper pole.

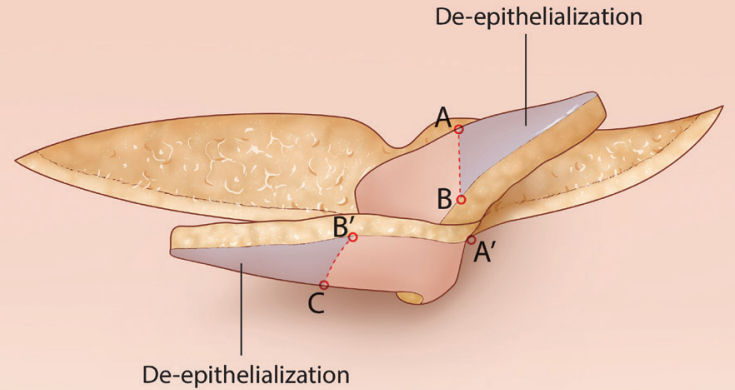

(B)

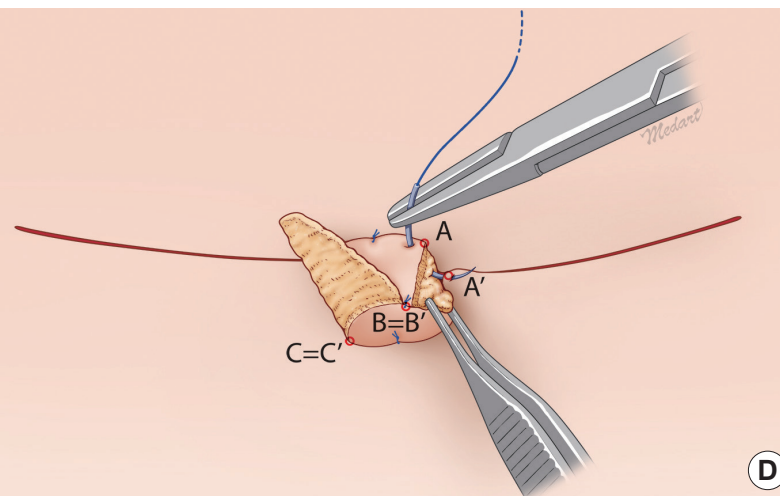

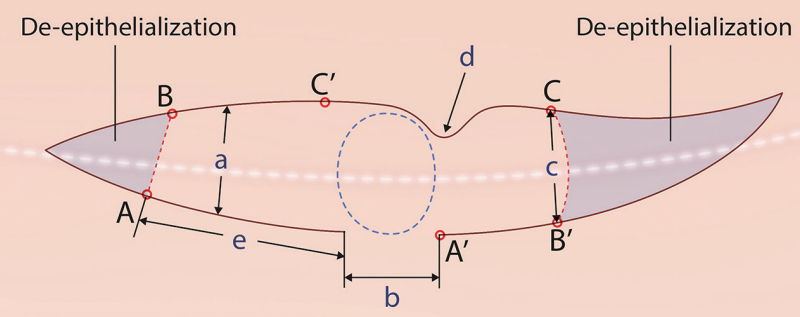

A

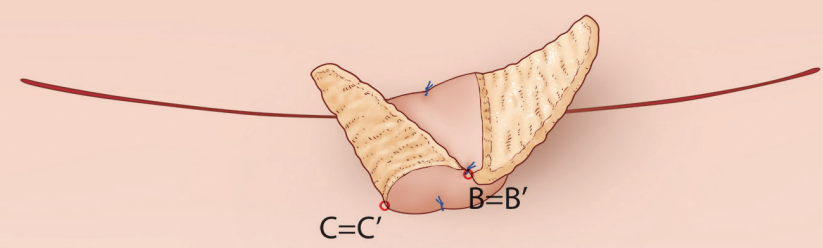

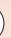

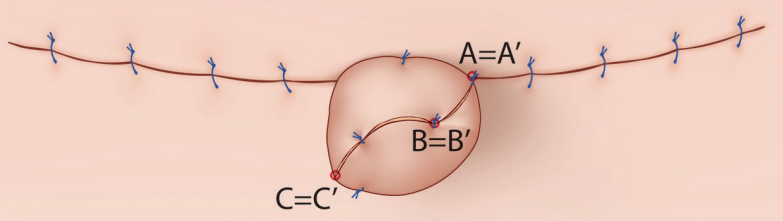


nipple is accordingly that of an upside-down quadrilateral and its base serves as a barrier so that the pillar may not easily sink into the breast mound (Fig. 2). Moreover, this method utilizes the previous mastectomy scar, thereby incorporating more connective tissue, and the nipple has denser structure, giving firmness to the pillar compared with the utilization of thin and friable dermal tissue in other local flap methods. Lastly, the lengthy mastectomy scar is utilized for nipple volume augmentation. During flap design, the 2 wings were designed within the length of the long scar, and excess tissue at the end of the wings was de-

\section{Fig. 2. A lateral view of the reconstructed nipple}

The cross-section of the nipple forms an upside-down quadrilateral with a narrower width at the base than at the top.

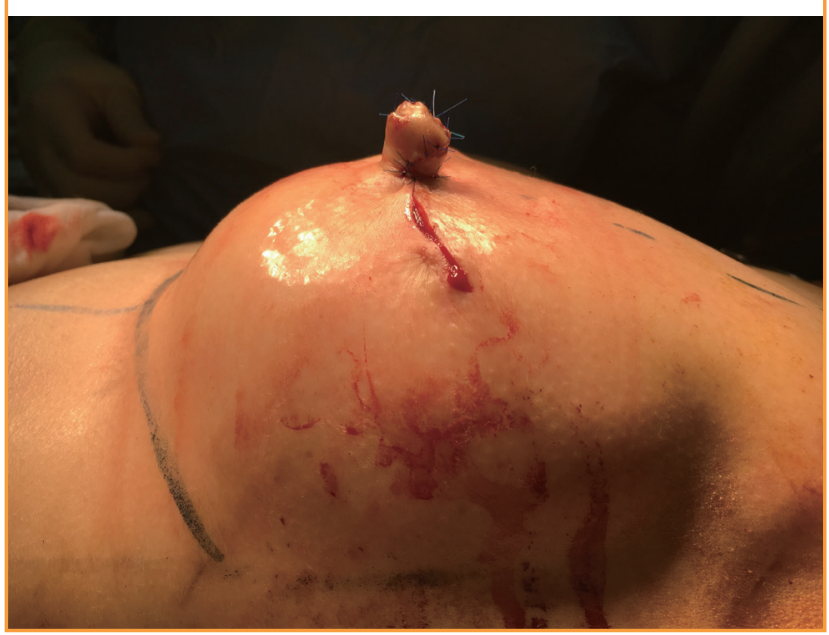

epithelialized and packed into the nipple.

With this novel boomerang flap, we successfully improved the maintenance of reconstructed nipple projection. Fig. 3 shows the case of a 53-year-old patient with well-maintained nipple projection 14 months postoperatively.

\section{DISCUSSION}

The fact that numerous nipple reconstruction methods have been developed implies an underlying dissatisfaction with the existing techniques. We have also struggled to find a convenient and reliable method for improving long-term nipple projection. First, we eliminated the $\mathrm{C}$ flap in the $\mathrm{C}-\mathrm{V}$ flap technique and used 1 wing to form the lid of the nipple, narrowing the base of the nipple compared to the upper part of the nipple. The crosssection of the newly formed nipple resembles that of an upsidedown trapezoid, and its base serves to prevent the pillar from easily sinking into the breast mound. Moreover, this technique, when possible, utilizes scar tissue as the nipple flap because it contains more connective tissue and is denser. Using scar tissue, rather than thin, friable dermal tissue, provides added firmness to the nipple pillar. Lastly, the lengthy scar remaining after the previous surgery was utilized to augment nipple volume. With this novel technique, we achieved improved preservation of the projection for the reconstructed nipple.

This is a simple and reliable method for nipple reconstruction that utilizes the previous surgical scar and maintains projection

\section{Fig. 3. A patient case of nipple reconstruction}

Medical photographs of a 53-year-old patient undergoing a left, skin-sparing mastectomy and two-stage breast reconstruction with simultaneous nipple reconstruction with a boomerang flap and right augmentation. Anterior- (A) and lateral-view (B) medical photographs 14 months after tissue expander removal and implant insertion, showing well-preserved long-term nipple projection.
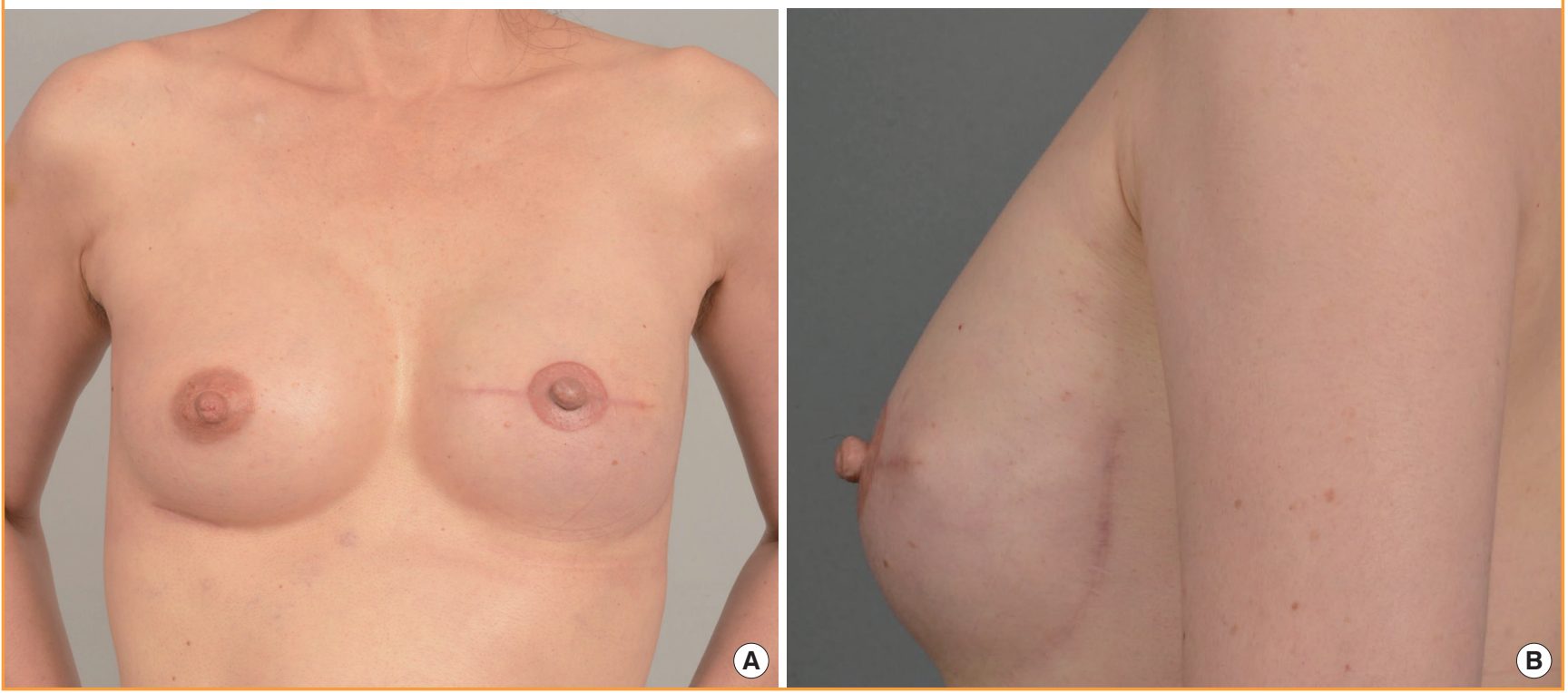
in the reconstructed nipple, and it can be widely applied. However, the previous literature has reported that nipple projection tends to decrease throughout the first 6 postoperative months. Further studies on nipple projection maintenance over a longterm follow-up period should be done to validate this method.

\section{REFERENCES}

1. Shestak KC, Gabriel A, Landecker A, et al. Assessment of long-term nipple projection: a comparison of three techniques. Plast Reconstr Surg 2002;110:780-6.
2. Zhong T, Antony A, Cordeiro P. Surgical outcomes and nipple projection using the modified skate flap for nipple-areolar reconstruction in a series of 422 implant reconstructions. Ann Plast Surg 2009;62:591-5.

3. Losken A, Mackay GJ, Bostwick J 3rd. Nipple reconstruction using the C-V flap technique: a long-term evaluation. Plast Reconstr Surg 2001;108:361-9.

4. Di Benedetto G, Sperti V, Pierangeli M, et al. A simple and reliable method of nipple reconstruction using a spiral flap made of residual scar tissue. Plast Reconstr Surg 2004;114: $158-61$.

Supplemental Video S1. One wing is rolled to form the pillar and the other wing, instead of a C flap, forms the lid, while 3 key sutures are made. The distal parts of the wings are de-epithelialized and packed into the nipple for core volume augmentation.

Supplemental data can be found at: http://e-aps.org/src/sm/aps-43-470-s001.avi 